\title{
MULTI MODAL MEDICAL IMAGE FUSION USING WEIGHTED LEAST SQUARES FILTER
}

\author{
B.S. Saini ${ }^{1}$, Vivek Venugopal ${ }^{2}$ \\ ${ }^{I}$ Associate Professor, ECE Dept., Dr B.R Ambedkar NIT Jalandhar, Jalandhar, Punjab, India \\ ${ }^{2}$ M.Tech Student, ECE Dept., Dr B.R Ambedkar NIT Jalandhar, Jalandhar, Punjab, India
}

\begin{abstract}
A novel multi modal medical image fusion method based on weighted least squares filter is proposed. To perform the image fusion, a two-scale decomposition of the input images is performed. Then weighted least squares filter is used to calculate the weight maps for the base and detail layers and then a weighted average of the base and detail layer is performed to obtain the fused image. The performance of the proposed method was compared with several other image fusion methods using five quality metrics based on information present $\left(Q_{M I}\right)$, structural information retained $\left(Q_{Y}\right.$ and $\left.Q_{C}\right)$, features retained $\left(Q_{G}\right.$ and $\left.Q_{P}\right)$ and it was found that the proposed method produces a robust performance for fusion of multi-modal images.
\end{abstract}

Keywords—Medical Image, Multimodality, Image Fusion, Weighted Least Squares Filter

\section{INTRODUCTION}

Medical imaging has advanced in the last few decades with the advent of various modalities of imaging such as CT (Computed Tomography), MRI (Magnetic Resonance Imaging) etc. Medical images are broadly classified, on the basis of modality, into structural images, which provide high resolution images with anatomical detail and precise localization capability, and functional images, which provide low resolution images with functional information which can be used for diagnosis purpose.[1] [2] The emergence of these multimodal medical images has prompted researchers to delve into a range of applications like classification, registration, denoising, fusion, etc.[3] Out of these applications, medical image fusion is the process of registering and combining complementary information present in 2 or more medical images which vary in modality there by providing a more complete and accurate description.

There has been extensive research in the field of medical image fusion and various image fusion algorithms have been devised for the same. Some of them include pixel level techniques like Principal Component Analysis (PCA), averaging, transform based techniques like wavelet transform, Multi-scale Geometric Analysis (MGA) techniques (contourlet, ripplet, etc), optimization based techniques like neural networks, fuzzy logic, etc. [4]-[7] pixel level techniques suffer from spectral degradation even though they provide better results. Wavelet transform cannot detect the smoothness along the edges. Moreover, wavelet transform provide limited directional information since wavelet decomposes image in only three directional highpass subbands, namely, vertical, horizontal and diagonal. This limits the ability to preserve the salient features of the source images and probably introduces some artifacts and inconsistency in the fused results. In the case of MGA techniques, it is not possible to measure the importance/contribution of individual source image in the fused image. Moreover, finding an effective way of combining the two source images is still an open problem.[2] Optimization based techniques are time consuming in nature since they require multiple iterations and they also tend to over smooth the edges which is not ideal as far as fusion is concerned. [8]

To solve the above mentioned problems, a novel method based on weighted least squares filter has been proposed in this paper. The remainder of the paper is organized as follows. Section II gives a brief insight on the weighted least squares filtering. Section III describes the proposed fusion algorithm. Section IV deals with the experimental results and discussions and conclusion is provided in section $\mathrm{V}$.

\section{WEIGHTED LEAST SQUARES FILTER}

Edge preserving filters like guided filter, bilateral filter and weighted least squares filter have been an active research topic in image processing since they do not blur the strong edges in the decomposition process there by reducing the ringing artifacts.

In edge preserving filtering, we try to find a new image $u$ from the input image $g$ which on one hand is as close as possible to $g$ and at the same time is as smooth as possible across everywhere except at places where there is a significant gradient in $g$. This can be seen as a minimization of the expression[9]

$\sum_{i=1}^{M} \sum_{j=1}^{N}(\mathrm{u}(\mathrm{i}, \mathrm{j})-g(\mathrm{i}, \mathrm{j}))^{2}+\lambda\left(\mathrm{a}_{\mathrm{x}, \mathrm{g}}(\mathrm{i}, \mathrm{j})\left(\frac{\partial u}{\partial x}\right)^{2}{ }_{i, j}+\mathrm{a}_{y, g}(\mathrm{i}, \mathrm{j})\left(\frac{\partial u}{\partial y}\right)^{2}{ }_{i, j}\right)$

where the first term in the summation is the data term, whose objective is to minimize the distance between $u$ and $g$. The second term in the summation is called as the regularization term, whose objective is to achieve smoothness by minimizing the partial derivative of $u$. $\lambda$ provides a balance between the two terms. Increasing the value of $\lambda$ leads to 
progressively smoother images. $\mathrm{a}_{\mathrm{x}, \mathrm{g}}(\mathrm{i}, \mathrm{j})$ and $\mathrm{a}_{y, g}(\mathrm{i}, \mathrm{j})$ are the smoothness weights along $\mathrm{x}$ and $\mathrm{y}$ and is dependent on $g$. It is given as

$$
\begin{aligned}
& \mathrm{a}_{\mathrm{x}, \mathrm{g}}(\mathrm{i}, \mathrm{j})=\left(\left|\frac{\partial l}{\partial x}\right|_{\mathrm{i}, \mathrm{j}}^{\alpha}+\varepsilon\right)^{-1} \\
& \mathrm{a}_{\mathrm{y}, \mathrm{g}}(\mathrm{i}, \mathrm{j})=\left(\left|\frac{\partial l}{\partial y}\right|_{i, \mathrm{j}}^{\alpha}+\varepsilon\right)^{-1}
\end{aligned}
$$

Where $l$ is the log-luminance channel of the input image $g, \alpha$ decides the sensitivity to the gradients of $g$, and $\varepsilon$ is a constant whose value is 0.0001 and comes of purpose where ever $g$ is constant.

\section{IMAGE FUSION WITH WEIGHTED LEAST SQUARES FILTER}

Fig.1. shows the flowchart of the proposed weighted least squares (WLS) filter based fusion method. Firstly, the input images are decomposed into two-scales by an average filter. The base layer of each input image is obtained by the formula[8]

$$
B_{n}=I_{n} * Z
$$

Where $I_{n}$ is the $n^{\text {th }}$ source image, $Z$ is the averaging filter of size $7 \times 7$. The detail layer is obtained by subtracting the base layer from the input image.

$$
D_{n}=I_{n}-B_{n}
$$

As a result of the two scale decomposition, we get a base layer which contains large scale variations in intensity and in detail layer, we get the small scale details.

To construct the weight map, a $3 \times 3$ Laplacian filter is applied to each input image to obtain the high pass image $\mathrm{H}_{n}$.

$$
H_{n}=I_{n} * L
$$

Next, the saliency map is constructed by passing the absolute value of $\mathrm{H}_{\mathrm{n}}$ through a Gaussian low pass filter of size $11 \mathrm{x} 11$. The standard deviation for the Gaussian filter is set at 5 .

$$
S_{n}=\left|H_{n}\right| * g_{r_{g}, \sigma_{g}}
$$

Where the parameters $r_{\mathrm{g}}$ and $\sigma_{\mathrm{g}}$ are the half window length and standard deviation of the Gaussian filter respectively. From the saliency map, the weight map is calculated as follows.

$$
P_{n}^{k}=\left\{\begin{array}{cc}
1 \text { if } \quad S_{n}{ }^{k}=\max \left(S_{1}{ }^{k}, S_{2}{ }^{k}, \ldots, S_{N}{ }^{k}\right) \\
0 & \text { Otherwise }
\end{array}\right.
$$

Where $\mathrm{N}$ is the number of input images, $S_{n}{ }^{k}$ is the saliency value for pixel $k$ in the $n$th image.

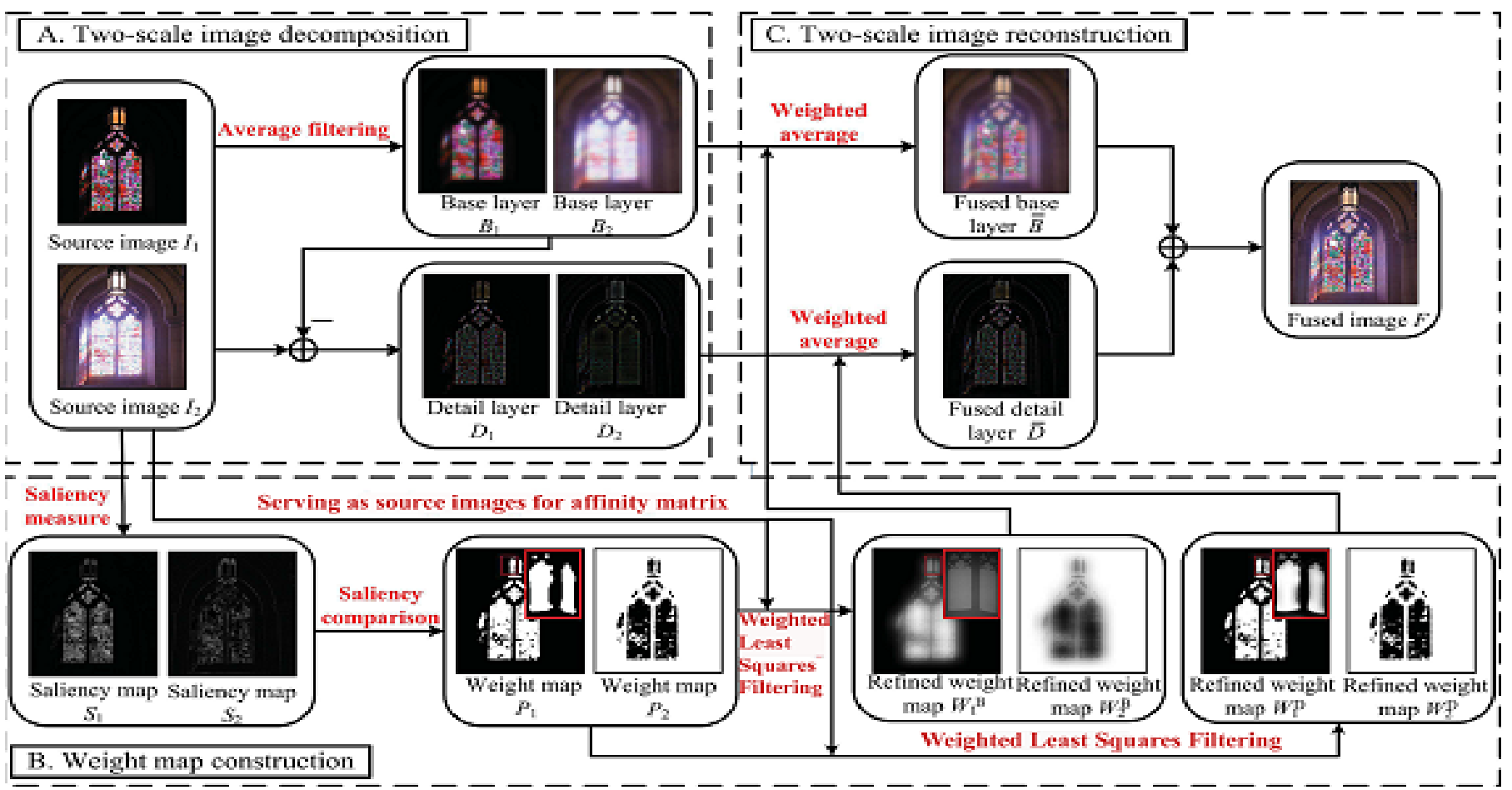

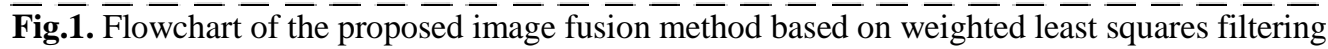


However, the weight maps obtained through the above process are usually noisy and are not aligned with the object boundaries. Therefore, we pass the weight maps through a weighted least squares filter for refining the weights and the resulting weights are normalized so that the sum of all weights for a particular pixel becomes one.

$$
\begin{aligned}
& W_{n}^{B}=W_{\lambda_{1}, \alpha_{1}}\left(\mathrm{P}_{\mathrm{n}}, \log \left(\mathrm{I}_{\mathrm{n}}\right)\right) \\
& W_{n}{ }^{D}=W_{\lambda_{2}, \alpha_{2}}\left(\mathrm{P}_{\mathrm{n}}, \log \left(\mathrm{I}_{\mathrm{n}}\right)\right)
\end{aligned}
$$

Where $\lambda_{1}, \alpha_{1}, \lambda_{2}, \alpha_{2}$ are the parameters of the weighted least squares filter. $W_{n}{ }^{B}$ and $W_{n}{ }^{D}$ are the refined weight maps for the base and detail layer respectively. Then, the base and detail layer of different input images are fused together by weighted averaging.

$$
\begin{aligned}
& \bar{B}=\sum_{n=1}^{N} W_{n}^{B} B_{n} \\
& \bar{D}=\sum_{n=1}^{N} W_{n}^{D} D_{n}
\end{aligned}
$$

The fused image is obtained by adding the fused base and detail layer.

$$
F=\bar{B}+\bar{D}
$$

\section{EXPERIMENTS AND DISCUSSION}

\subsection{Experimental Setup}

For the comparison of the proposed technique with other existing image fusion algorithms, an image database of 10 pairs of PET and MRI images of the head from the Harvard brain atlas database is considered. The PET image provides details pertaining to the functional aspect of the brain, in this case regarding whether the person is suffering from mild Alzheimer's disease or not and the MRI image provides details pertaining to the structural aspect of the brain.
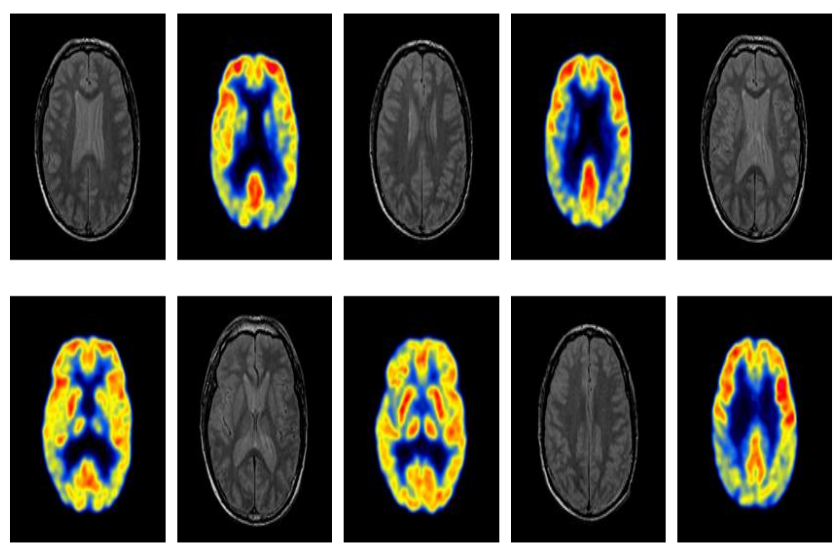

Fig.2. Sample Images of the multi-modal image database

Fig.2. shows some of the images of the multi-modal image database. The proposed Weighted Least Squares filter based fusion (WLS) has been compared with eight other image fusion algorithms based on Laplacian pyramid (LAP)[10], shift invariant wavelet transform (SWT)[11], curvelet transform (CVT)[12], non subsampled contourlet transform (NSCT), generalized random walks (GRW)[13], waveletbased statistical sharpness measure (WSSM)[14] and higher order singular value decomposition (HOSVD)[15] respectively. The parameter settings for the above mentioned methods have been obtained from [2] and for the WLS based fusion the value of $\lambda$ and $\alpha$ are 3 and 5 for the base layer images and 0.1 and 10 for the detail layer images.

\subsection{Image Fusion Quality Metrics}

In order to compare the performance of different fusion algorithms with the proposed WLS based fusion, 5 different quality metrics based on information present $\left(\mathrm{Q}_{\mathrm{MI}}\right)$, structural information retained $\left(\mathrm{Q}_{\mathrm{Y}}\right.$ and $\left.\mathrm{Q}_{\mathrm{C}}\right)$, features retained $\left(\mathrm{Q}_{\mathrm{G}}\right.$ and $\mathrm{Q}_{\mathrm{P}}$ ) have been considered.

\subsubsection{Normalized Mutual Information $\left(Q_{M I}\right)$}

Traditional mutual information based quality metrics suffer from being unstable and also bias the measure towards the source image with the highest entropy. Therefore Hossny et al[16] devised a normalized mutual information based quality metric to measure how well the information from the source images is preserved in the fused image. It is given by the formula

$$
\mathrm{Q}_{\mathrm{MI}}=2\left[\frac{\mathrm{MI}(\mathrm{A}, \mathrm{F})}{\mathrm{H}(\mathrm{A})+\mathrm{H}(\mathrm{F})}+\frac{\mathrm{MI}(\mathrm{B}, \mathrm{F})}{\mathrm{H}(\mathrm{B})+\mathrm{H}(\mathrm{F})}\right]
$$

Where $\mathrm{MI}(\mathrm{A}, \mathrm{F})$ is the mutual information between input image $A$ and fused image $F$ and $H(A)$ and $H(F)$ are the entropy of $\mathrm{A}$ and $\mathrm{F}$ respectively. The mutual information between 2 images is given by the formula

$$
\mathrm{MI}(\mathrm{A}, \mathrm{F})=\mathrm{H}(\mathrm{A})+\mathrm{H}(\mathrm{F})-\mathrm{H}(\mathrm{A}, \mathrm{F})
$$

Where $\mathrm{H}(\mathrm{A}, \mathrm{F})$ is the joint entropy of images $\mathrm{A}$ and $\mathrm{F}$. The larger the value of the $\mathrm{Q}_{\mathrm{MI}}$, better the quality of the image resulting from fusion.

\subsubsection{Yang et. al's Quality Metric $\left(Q_{\mathrm{Y}}\right)$}

Yang's metric[17] measures how well the structural information of the source images is preserved in the fused image.It is mathematically defined as

$Q_{Y}=\left\{\begin{array}{l}\lambda_{w} \operatorname{SSIM}\left(A_{w}, F_{w}\right)+\left(1-\lambda_{w}\right) \operatorname{SSIM}\left(B_{w}, F_{w}\right), \operatorname{if} \operatorname{SSIM}\left(A_{w}, B_{w} \mid w\right) \geq 0.75 \\ \max \left\{\operatorname{SSIM}\left(A_{w}, F_{w}\right), \operatorname{SSIM}\left(B_{w}, F_{w}\right)\right\}, \text { if } \operatorname{SSIM}\left(A_{w}, B_{w} \mid w\right)<0.75\end{array}\right.$

Where $\mathrm{A}$ and $\mathrm{B}$ are the input images and $\mathrm{F}$ is the fused image, $w$ is a window of size $7 \times 7, \lambda_{w}$ is the local weight given by the formula 


$$
\lambda_{w}=\frac{s\left(\mathrm{~A}_{\mathrm{w}}\right)}{s\left(\mathrm{~A}_{\mathrm{w}}\right)+s\left(B_{\mathrm{w}}\right)}
$$

Where $s\left(A_{w}\right)$ and $s\left(B_{w}\right)$ gives the variance of the input images $A$ and $B$ within the window w respectively. SSIM is the structural similarity index given by

$$
\operatorname{SSIM}\left(\mathrm{A}_{\mathrm{w}}, \mathrm{B}_{\mathrm{w}}\right)=E\left(\frac{\left(2 \mu_{A_{w}}+\mu_{B_{w}}+c_{1}\right)\left(2 \sigma_{\mathrm{d}}+\mathrm{c}_{2}\right)}{\left(\left(\mu_{A_{w}}\right)^{2}+\left(\mu_{B_{w}}\right)^{2}+c_{1}\right)\left(\left(\sigma_{A_{w}}\right)^{2}+\left(\sigma_{B_{w}}\right)^{2}+\mathrm{c}_{2}\right)}\right)
$$

Where $\mathrm{c}_{1}$ and $\mathrm{c}_{2}$ are constants, $\mu_{A_{w}}$ and $\mu_{B_{w}}$ are the mean pixel intensity values of input images $\mathrm{A}$ and $\mathrm{B}$ in the window $\mathrm{w}, \sigma_{A_{w}}$ and $\sigma_{B_{w}}$ are the standard deviation of images $\mathrm{A}$ and $\mathrm{B}$ in the window $\mathrm{w}$ and $\sigma_{\mathrm{d}}$ is the covariance of $A$ and $B$ in the window $w$. The larger the value of the $\mathrm{Q}_{\mathrm{Y}}$, better the quality of the image resulting from fusion.

\subsubsection{Cjevic et. al's Quality Metric $\left(Q_{C}\right)$}

Cjevic's metric[18] estimates how well the important information in the source images is preserved in the fused image.It is given by the formula

$$
Q_{C}=\mu\left(\mathrm{A}_{\mathrm{w}}, B_{\mathrm{w}}, F_{\mathrm{w}}\right) \mathrm{UIQI}\left(\mathrm{A}_{\mathrm{w}}, F_{\mathrm{w}}\right)+\left(1-\mu\left(\mathrm{A}_{\mathrm{w}}, B_{\mathrm{w}}, F_{\mathrm{w}}\right)\right) \mathrm{UIQI}\left(B_{\mathrm{w}}, F_{\mathrm{w}}\right)
$$

Where $\mu\left(A_{w}, B_{w}, F_{w}\right)$ is the local weight in a window $w$ given by the formula:

$$
\mu\left(\mathrm{A}_{\mathrm{w}}, B_{\mathrm{w}}, F_{\mathrm{w}}\right)=\left\{\begin{array}{lc}
0 & \text { if } \frac{\sigma_{A F}}{\sigma_{A F}+\sigma_{B F}}<0 \\
\frac{\sigma_{A F}}{\sigma_{A F}+\sigma_{B F}} & \text { if } 0<\frac{\sigma_{A F}}{\sigma_{A F}+\sigma_{B F}}<1 \\
1 & \text { if } \frac{\sigma_{A F}}{\sigma_{A F}+\sigma_{B F}}>1
\end{array}\right.
$$

Where $\sigma_{A F}$ and $\sigma_{B F}$ are the covariance of images $\mathrm{A}$ and $\mathrm{B}$ with $\mathrm{F}$ respectively and $\operatorname{UIQI}\left(\mathrm{A}_{\mathrm{w}}, \mathrm{F}_{\mathrm{w}}\right)$ is the universal image quality index between $\mathrm{A}$ and $\mathrm{F}$ in the window w. UIQI is calculated as follows.

$$
\operatorname{UIQI}\left(\mathrm{A}_{\mathrm{w}}, F_{\mathrm{w}}\right)=\frac{4 \sigma_{A F} \mu_{A} \mu_{F}}{\left(\sigma_{\mathrm{A}}+\sigma_{F}\right)\left(\mu_{\mathrm{A}}^{2}+\mu_{F}^{2}\right)}
$$

Where $\sigma_{A F}$ is the covariance of image A with $\mathrm{F}, \mu_{A}$ and $\mu_{F}$ are the average pixel intensity values of images $\mathrm{A}$ and $\mathrm{F}$ and $\sigma_{\mathrm{A}}, \sigma_{F}$ are the standard deviation of images $\mathrm{A}$ and $\mathrm{F}$ respectively. The larger the value of the $\mathrm{Q}_{\mathrm{C}}$, better the quality of the image resulting from fusion.

\subsubsection{Gradient Based Index $\left(Q_{G}\right)$}

The gradient based index $\left(\mathrm{Q}_{\mathrm{G}}\right)[19]$ measures how well the edge information of the source images is preserved and is given by the formula provided in equation number 22 .

$$
Q_{G}=\frac{\sum_{i=1}^{M} \sum_{j=1}^{N}\left(\mathrm{Q}_{\mathrm{AF}}(\mathrm{i}, \mathrm{j}) \tau^{\mathrm{A}}(\mathrm{i}, \mathrm{j})+\mathrm{Q}_{B \mathrm{~F}}(\mathrm{i}, \mathrm{j}) \tau^{B}(\mathrm{i}, \mathrm{j})\right)}{\sum_{i=1}^{M} \sum_{j=1}^{N}\left(\tau^{\mathrm{A}}(\mathrm{i}, \mathrm{j})+\tau^{B}(\mathrm{i}, \mathrm{j})\right)}
$$

Where the image is considered of size $\mathrm{MxN}, \mathrm{Q}_{\mathrm{AF}}(\mathrm{i}, \mathrm{j})$ and $\mathrm{Q}_{\mathrm{BF}}(\mathrm{i}, \mathrm{j})$ are the edge strength at pixel location $(\mathrm{i}, \mathrm{j})$ and $\tau^{\mathrm{A}}(\mathrm{i}, \mathrm{j})$ and $\tau^{B}(\mathrm{i}, \mathrm{j})$ denote the importance of $\mathrm{Q}_{\mathrm{AF}}(\mathrm{i}, \mathrm{j})$ and $\mathrm{Q}_{\mathrm{BF}}(\mathrm{i}, \mathrm{j})$ respectively. The larger the value of the $\mathrm{Q}_{\mathrm{G}}$, better the quality of the image resulting from fusion.

\subsubsection{Phase Congruency Based Index $\left(Q_{P}\right)$}

$\mathrm{Q}_{\mathrm{P}}$ [8]measures how well the important features present in the input images are preserved in the fused image and is given by the formula

$$
Q_{P}=\left(P_{p}\right)^{\alpha}\left(\mathrm{P}_{\mathrm{M}}\right)^{\beta}\left(\mathrm{P}_{\mathrm{m}}\right)^{\gamma}
$$

Where $\mathrm{P}_{\mathrm{p}}, \mathrm{P}_{\mathrm{M}}, \mathrm{P}_{\mathrm{m}}$ are the phase congruency, maximum and minimum moment parameters respectively. $\alpha, \beta$ and $\gamma$ are the exponents and is set to one in this paper

\subsection{Experimental Results and Discussions}

Fig 3 shows sample of two medical images from the multimodal medical image database, Magnetic Resonance Imaging (MRI) and Positron Emission Tomography (PET) of a patient suffering from mild Alzheimer's disease respectively. The MRI image shows the structure and the PET image shows the metabolic activity present inside the human head. Table I shows the result of the fusion of the two images using the proposed method and other exisitng image fusion algorithms. The result produced by SWT and GRW method reduces the brightness of the overall fused image there by making some details unclear. The WSSM based method does not work for this set of images because it introduces serious artifacts in the fused image. The HOSVD based method reduces the brightness in the metabolic information there by losing some important metabolic information. Though, the GFF and WLS based fusion algorithms are able to preserve the features of the input images without any visible artifacts and brightness distortions, and are also able to preserve the complementary information present in both the input images. 

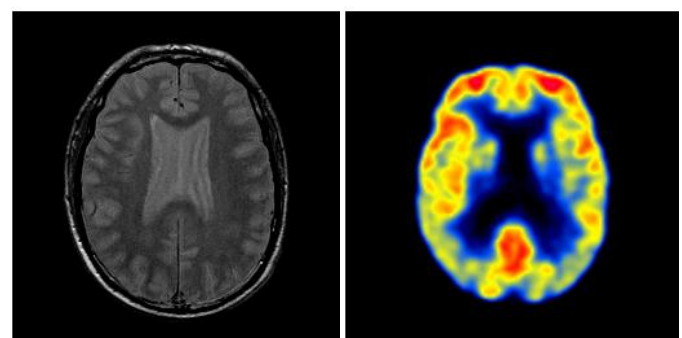

Fig.3. Sample input images

Table 2 provides an objective performance of different methods. The value of each parameter is calculated for each pair of images in the database and the average value is displayed in Table 3. It can be seen that though HOSVD based fusion method has the maximum average $\mathrm{Q}_{\mathrm{MI}}$ values in the database, it provides a relatively poor performance in terms of $\mathrm{Q}_{\mathrm{C}}$ (second worst), $\mathrm{Q}_{\mathrm{P}}$ (fourth worst)and $\mathrm{Q}_{\mathrm{G}}$ (fourth worst)in the database. Higher value of $\mathrm{Q}_{\mathrm{MI}}$ means that the original information present in the different input images is preserved in the fused image. But a higher value of $\mathrm{Q}_{\mathrm{MI}}$ can also occur if the fused image is closer to one of the input images. Therefore all five quality metrics need to be considered together while comparing the fusion performance of each method. The WLS based fusion technique, in comparison, though may not always be the best in all five quality metrics, but it has a very stable performance(always within top two). Thus, it can be shown that the proposed method can achieve state of the art fusion performance in the case of multi-modal image fusion.

Table 1 Output of different image fusion algorithms for multi-modal medical images

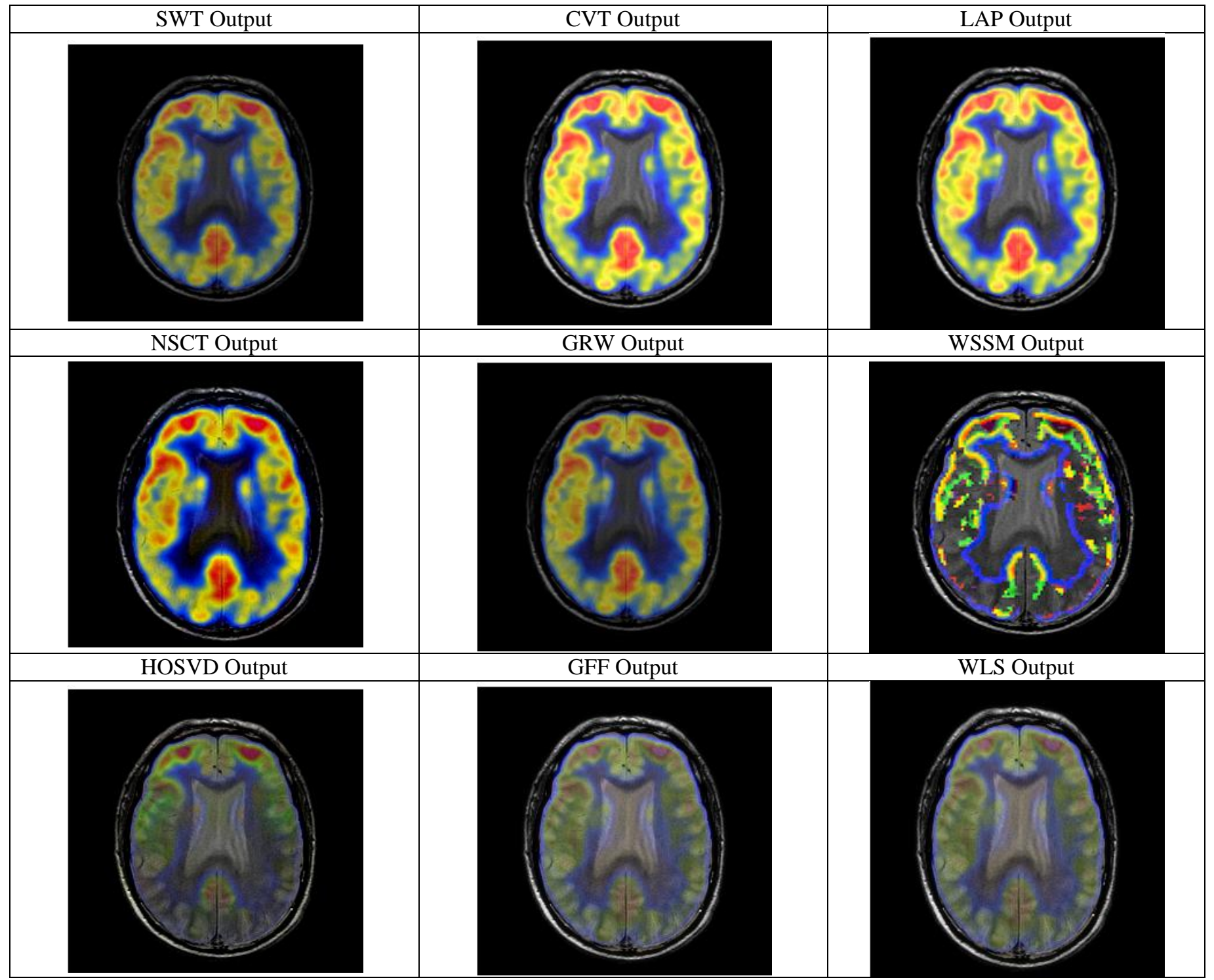


Table 2 Objective performances of different image fusion methods

\begin{tabular}{|l|l|l|l|l|l|l|l|l|l|l|}
\hline $\begin{array}{l}\text { Source } \\
\text { Images }\end{array}$ & Index & SWT & CVT & LAP & NSCT & GRW & WSSM & HOSVD & GFF & WLS \\
\hline \multirow{4}{*}{$\begin{array}{l}\text { Harward } \\
\begin{array}{l}\text { Medical } \\
\text { Database }\end{array}\end{array}$} & $\mathrm{Q}_{\mathrm{Y}}$ & 0.6975 & 0.7019 & 0.7621 & 0.7685 & 0.6851 & 0.7914 & 0.9133 & 0.8943 & $\mathbf{0 . 9 2 8 1}$ \\
\cline { 2 - 11 } & $\mathrm{Q}_{\mathrm{C}}$ & 0.6108 & 0.6470 & 0.6645 & 0.6815 & 0.5425 & 0.7132 & 0.6085 & 0.7734 & $\mathbf{0 . 8 0 9 2}$ \\
\cline { 2 - 11 } & $\mathrm{Q}_{\mathrm{G}}$ & 0.5752 & 0.5481 & 0.6670 & 0.6384 & 0.4429 & 0.6193 & 0.6006 & 0.6802 & $\mathbf{0 . 7 0 4 3}$ \\
\cline { 2 - 11 } & $\mathrm{Q}_{\mathrm{P}}$ & 0.5215 & 0.4722 & 0.5742 & 0.5443 & 0.4050 & 0.3392 & 0.5182 & 0.6310 & $\mathbf{0 . 6 7 4 3}$ \\
\cline { 2 - 11 } & $\mathrm{Q}_{\mathrm{MI}}$ & 0.5754 & 0.5192 & 0.5302 & 0.6700 & 0.5614 & 0.7136 & $\mathbf{0 . 8 6 0 8}$ & 0.6848 & 0.7222 \\
\hline
\end{tabular}

\section{CONCLUSIONS}

In this paper, a novel image fusion method based on weighted least squares filter was presented for multi-modal medical image fusion. The proposed method uses a twoscale representation using an average filter. The weighted least squares filter is used in a novel way for refinining the weight maps of the base and detail layers. Experiments show that the proposed method can preserve the complementary information present in multiple input images without introducing any artifacts or distortions. The proposed method also gives a robust performance in terms of the different quality metrics. Future work in this area could be setting the different parameters of the weighted least squares in adaptively to improve the performance of the proposed method

\section{REFERENCES}

[1] S. Das, M. Chowdhury, M. K. Kundu "Medical Image Fusion Based On Ripplet Transform Type-I", Progress In Electromagnetics Research B, Vol. 30, 355-370, 2011

[2] S. Das, M.K Kundu "A Neuro-Fuzzy Approach for Medical Image Fusion", IEEE Transactions On Biomedical Engineering, Vol. 60, 3347-3353,2013

[3] R. Singh , R. Srivastava, O. Prakash, A. Khare. "Mixed Scheme Based Multimodal Medical Image Fusion using Daubechies Complex Wavelet Transform", IEEE/OSA/IAPR International Conference on Informatics, Electronics \& Vision 2012

[4] C. He, Q. Liu, H. Li, H. Wang, "Multimodal Medical Image Fusion Based on IHS and PCA", Symposium on Security Detection and Information Processing, 2010.

[5] Q. Guihong, Z. Dali, Y. Pingfan, “ Medical Image Fusion by Wavelet Transform Modulus Maxima", Optics Express 9 (4), 184-190, 2001.

[6] L. Yang, B.L. Guo, W. Ni, and Y. Tagawa, "Multimodality Medical Image Fusion Based on Multiscale Geometric Analysis of Contourlet Transform," Neurocomputing 72,203-211,2008

[7] A. Villeger, L. Ouchchane, J.J Lemaire, J.Y Boire, “ Data Fusion and Fuzzy Spatial Relationships for Locating Deep Brain Stimulation Targets in Magnetic Resonance Images " in Advanced Concepts for Intelligent Vision Systems, Springer, 909-919, 2006

[8] S. Li, X. Kang, S. Member, and J. Hu, "Image Fusion with Guided Filtering," vol. 22, no. 7, pp. 28642875, 2013.

[9] Z. Farbman, D. Lischinski, and R. Szeliski, "EdgePreserving Decompositions for Multi-Scale Tone and Detail Manipulation," 2007.

[10] P. Burt and E. Adelson, "The Laplacian pyramid as a compact image code," Commun. IEEE Trans., vol. C, no. 4, pp. 532-540, 1983.

[11] O. Rockinger, "Image Sequence Fusion Using a Shift-Invariant Wavelet Transform," pp. 288-291, 1997.

[12] F. E. Ali, A. A. Saad, and F. E. A. El-samie, "Fusion of MR and CT Images Using The Curvelet Transform," no. Nrsc, 2008.

[13] R. Shen, I. Cheng, and J. Shi, "Generalized Random Walks for Fusion of Multi-exposure images," vol. 20, no. 12, pp. 1-13, 2011.

[14] J. Tian and L. Chen, "Adaptive multi-focus image fusion using a wavelet-based statistical sharpness measure," Signal Processing, vol. 92, no. 9, pp. 2137-2146, 2012.

[15] Junli Liang, Yang He, Ding Liu, and Xianju Zeng, "Image Fusion Using Higher Order Singular Value Decomposition," vol. 21, no. 5, pp. 2898-2909, 2012.

[16] M. Hossny, S. Nahavandi, and D. Creighton, "Comments on " Information measure for performance of image fusion ,"' vol. 44, no. 18, pp. 2-3, 2008.

[17] C. Yang, J.-Q. Zhang, X.-R. Wang, and X. Liu, “A novel similarity based quality metric for image fusion," Inf. Fusion, vol. 9, no. 2, pp. 156-160, Apr. 2008.

[18] N. Cvejic, A. Łoza, D. Bull, and N. Canagarajah, “A Similarity Metric for Assessment of Image Fusion Algorithms," vol. 1, no. 4.

[19] C.S. Xydeas, V. Petrovic, "Objective Image Fusion Performance Measure," Electronics Letters vol. 36, no. 4, pp. 308-309, 2000. 